Sādhanā Vol. 37, Part 6, December 2012, pp. 637-655. (C) Indian Academy of Sciences

\title{
Reducing friction-induced vibration using intelligent active force control (AFC) with piezoelectric actuators
}

\author{
S M HASHEMI-DEHKORDI ${ }^{1, *}$, A R ABU-BAKAR ${ }^{2}$ and \\ M MAILAH ${ }^{3}$ \\ ${ }^{1}$ Department of Applied Mechanics, ${ }^{2}$ Department of Automotive Engineering and \\ ${ }^{3}$ Department of Applied Mechanics, Faculty of Mechanical Engineering, \\ Universiti Teknologi Malaysia, 81310 Skudai, Johor, Malaysia \\ e-mail: s.mahdi.hashemi.d@gmail.com; arahim@fkm.utm.my; \\ musa@fkm.utm.my
}

MS received 23 January 2011; revised 11 August 2012; accepted 12 October 2012

\begin{abstract}
In this paper, a novel approach to reduce the effect of mode coupling that causes friction induced vibration (FIV) is proposed by applying an intelligent active force control (AFC)-based strategy employing piezoelectric actuators with hysteresis effect to a simplified two degree-of-freedom mathematical model of a friction-induced vibration system. At first, the model is simulated and analysed using a closed loop pure Proportional-Integral-Derivative (PID) controller. Later, it is integrated with the intelligent AFC with fuzzy logic (FL) estimator and simulated under similar operating condition. After running several tests with different sets of operating and loading conditions, the results both in time and frequency domains show that the PID controller with the intelligent AFC is much more effective in reducing the vibration, compared to the pure PID controller alone.
\end{abstract}

Keywords. Friction-induced vibration; mode coupling; active force control; fuzzy logic estimator; piezoelectric actuators.

\section{Introduction}

Most of the mechanical systems that have sliding surfaces such as brake systems, clutches, saws, computer discs, position control systems and so on suffered from friction-induced vibration (FIV). Thus, it should be reduced or controlled as much as possible in order to achieve quieter vehicle (brake system), better performance (position control systems and clutches) and quality products.

There were typically three major mechanisms that contributing to the generation of friction induced vibration namely: negative damping (Shin et al 2004), sprag slip (Earles \& Lee 1976)

*For correspondence 
and modal coupling (Akay et al 2000). Various techniques such as structural modification and control system had been implemented in the work of the previous researchers to reduce the effect of FIV. The following will explain some of the recent work that had been done for reducing FIV. Ouyang et al (2009) presented structural modification approach in order to reduce vibration using complex eigenvalue analysis. They determined the latent roots of asymmetric systems represented by second order matrix differential equations and predicting the critical value of the parameter generating the asymmetry in the stiffness matrix, based on the receptance of the corresponding symmetric systems. Nakano \& Maegawa (2009) introduced safety-design criteria to prevent friction-induced vibration by conducting dimensionless analysis and numerical simulation for a single-degree-of-freedom system with friction. The model considered a discontinuity between static and kinetic friction and the dependence of the kinetic friction coefficient on the relative velocity. Due to dimensionless description, the number of parameters reduced from nine to five, where four of the five dimensionless parameters controlled the occurrence limit of friction-induced vibration. The occurrence-limit equation was derived on the basis of a previous study on stick-slip in the Coulomb friction model, and the discriminant inequalities were constructed with the four parameters in which they are sufficient for preventing friction-induced vibration. Chatterjee \& Mahata (2009) employed an active absorber based on the time-delayed displacement difference feedback in controlling friction-driven vibrations. The local stability analysis showed that the static equilibrium can be locally stabilized by suitably selecting the control gain and the time-delay. The regions of stability were delineated in the plane of the control parameters. Numerical simulations of the system showed that proper selections of the control parameters can also achieve the global stability of the system. As mentioned before, FIV was one of the major reasons that led to disk brake noise, and it was so important that as noted by Abendroth \& Wernitz (2000), a large number of manufacturers of brake pad materials spent up to $50 \%$ of their engineering costs on issues related to noise, vibration and harshness. Brake noise vibration phenomena were described by a number of terminologies and sometimes interchangeably used such as squeal, groan, chatter, judder, moan and hum (Kinkaid et al 2003). Apart from those mechanisms, moving load (Ouyang et al 1998) was another mechanism that led to disk brake noise. A detailed explanation of these mechanisms can be found in Kinkaid et al (2003).

Since then, problem of the noise and vibration in brake had been studied with experimental (Fieldhouse \& Newcomb 1993), analytical (Shin et al 2004) and finite element methods (Papinniemi et al 2002). Yet, no single method is available to fully suppress the entire brake noise and vibration in general and squeal in particular. Furthermore, a complete understanding on the problem had still not been achieved. Papinniemi et al (2002) suggested that these were mainly due to the complexity of the mechanisms itself and competitive nature of automotive industry which limits the amount of cooperative research, i.e., published in the open literature or public domain.

In this paper, a closed loop control employing PID element with and without active force control (AFC) was presented. These controllers were integrated into a friction induced vibration mathematical model that had two degrees-of-freedom and it was based on the mode coupling effect. The main advantage of the AFC technique was its ability to reject disturbances that were applied to the system through appropriate manipulation of the selected parameters. Additionally, the technique required much less computational burden and had been successfully established to be readily implemented in real-time. AFC as first proposed by Hewit \& Burdess (1981) was robust and effective in controlling a robot arm. Mailah \& Rahim (2000), Priyandoko et al (2009), Hashemi-Dehkordi et al (2009a, b, 2010) demonstrated the application of the technique to include many other dynamical systems with the incorporation of artificial intelligence (AI) methods. The effect of hysteresis in piezoelectric actuators was simulated, in order to provide the 
required force for reducing the vibrations. Although piezoelectric actuators with hysteresis had been widely studied and modelled (U-Xuan et al 2009), but in this paper, a simple simulation of it was carried out based on some considered assumptions.

\section{The friction-induced vibration model}

In order to take into account the effect of mode coupling, Hoffmann model (Hoffmann et al 2004) is adopted and is shown in figure 1. The model has two DOFs and consists of a conveyor belt with constant velocity $v_{\mathrm{B}}$ that is pushed with a constant normal force $\boldsymbol{N}$ against a block modelled as a block of mass $m$. The model has a single-point mass sliding over a conveyor belt and there are two linear springs $k_{1}$ and $k_{2}$ parallel and normal to the belt surface with the latter regarded as the physical contact stiffness between the objects in relative sliding motion. In addition, there is another linear spring $k$ mounted at oblique angle of $45^{\circ}$ constituting the off-diagonal terms in the model's stiffness matrix. For the friction component, a coulomb model is assumed such that $F_{\mathrm{F}}=\mu \mathrm{N}$, where $\mu$ is the coefficient of kinetic friction usually taken to be constant. $N$ is a normal force and since the normal force at the friction interface is linearly related to the vertical displacement $x_{2}$ of the mass then the resulting friction will become $F_{\mathrm{F}}=\mu k_{2} x_{2}$.

The matrix form of the equation of motion can be expressed as (Hoffmann et al 2004):

$$
\begin{gathered}
{\left[\begin{array}{cc}
m & 0 \\
0 & m
\end{array}\right]\left(\begin{array}{l}
\ddot{x}_{1} \\
\ddot{x}_{2}
\end{array}\right)+\left[\begin{array}{cc}
c_{1} & 0 \\
0 & c_{2}
\end{array}\right]\left(\begin{array}{c}
\dot{x}_{1} \\
\dot{x}_{2}
\end{array}\right)+\left[\begin{array}{cc}
k_{1}+\frac{k}{2} & \frac{-k}{2} \\
\frac{-k}{2} & k_{2}+\frac{k}{2}
\end{array}\right]\left(\begin{array}{l}
x_{1} \\
x_{2}
\end{array}\right)} \\
\quad=\left(\begin{array}{c}
-\mu k_{2} x_{2} \operatorname{sgn}\left(v_{\mathrm{B}}-\dot{x}_{1}\right) \\
N
\end{array}\right) .
\end{gathered}
$$

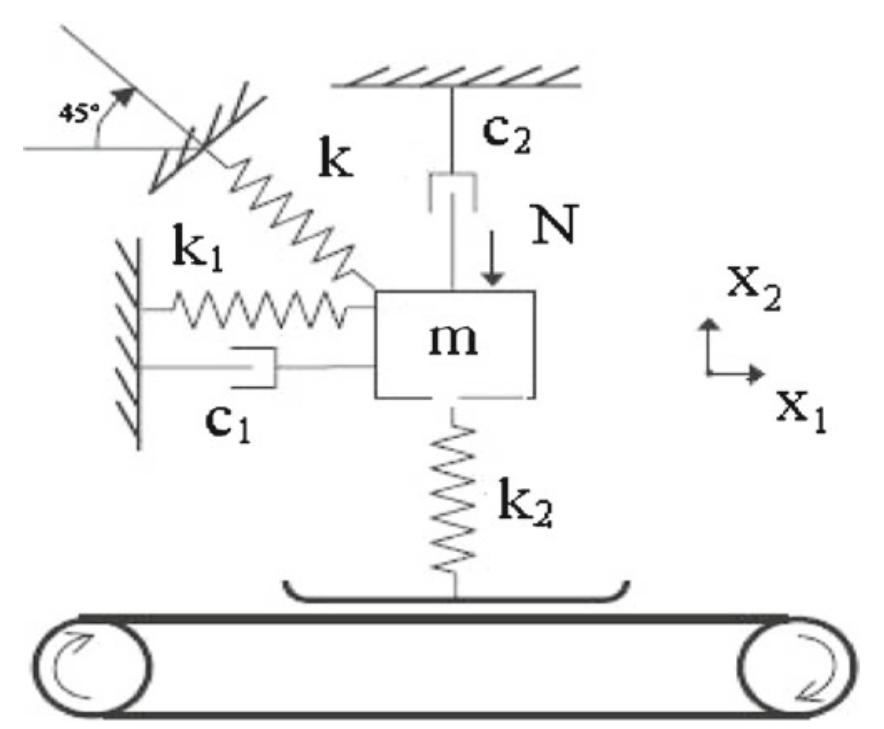

Figure 1. Hoffmann model of friction-induced vibration. 


\section{Control strategy}

Upon obtaining the model of the friction-induced vibration and its related equations of motion, it is required to control the vibration in both directions $x_{1}$ and $x_{2}$, by adding actuators which can produce forces that are parallel to the given axes. Thus, the equations of motion after applying the actuators can be obtained as follows

$$
\begin{gathered}
{\left[\begin{array}{cc}
m & 0 \\
0 & m
\end{array}\right]\left(\begin{array}{l}
\ddot{x}_{1} \\
\ddot{x}_{2}
\end{array}\right)+\left[\begin{array}{cc}
c_{1} & 0 \\
0 & c_{2}
\end{array}\right]\left(\begin{array}{l}
\dot{x}_{1} \\
\dot{x}_{2}
\end{array}\right)+\left[\begin{array}{cc}
k_{1}+\frac{k}{2} & \frac{-k}{2} \\
\frac{-k}{2} & k_{2}+\frac{k}{2}
\end{array}\right]\left(\begin{array}{l}
x_{1} \\
x_{2}
\end{array}\right)} \\
=\left(\begin{array}{c}
-\mu k_{2} x_{2} \operatorname{sgn}\left(v_{\mathrm{B}}-\dot{x}_{1}\right)+A_{1} \\
N+A_{2}
\end{array}\right),
\end{gathered}
$$

where forces $A_{1}$ and $A_{2}$ are the actuator forces that are applied to the mathematical model. A control strategy is proposed here employing an active force control (AFC) based scheme that is used in conjunction with the conventional PID controller. The PID controller is tuned with Ziegler-Nichol's method and then the obtained values are manipulated with trial and error for better performance. Later, the AFC part is incorporated into the system to provide the compensation of the disturbances that are inherent in the FIV system. Figure 2 shows the AFC scheme applied to a dynamic translation system. AFC scheme is shown to be very effective provided that the actuated force and body acceleration are accurately measured and at the same time the estimated mass property appropriately approximated (Mailah \& Rahim 2000). The essential AFC equation can be related to the computation of the estimated disturbance force, $F_{\mathrm{d}}$ as follows:

$$
F_{\mathrm{d}}=F-M^{\prime} \cdot a,
$$

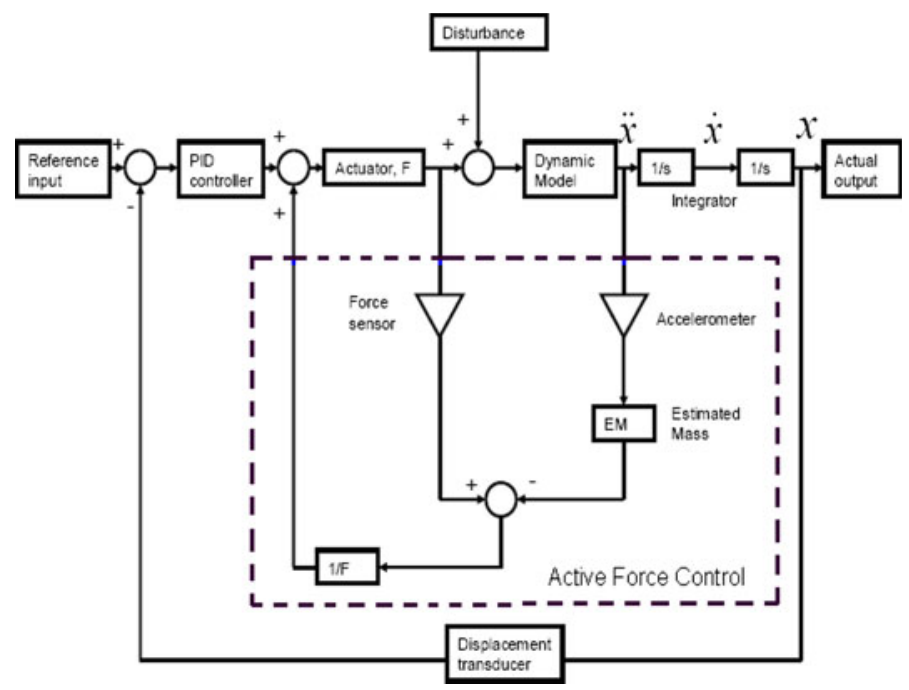

Figure 2. Schematic diagram of AFC strategy. 
where $F$ is the measured actuating force, $M^{\prime}$ is the estimated mass and $a$ is the measured linear acceleration. This parameter is then fed back through a suitable inverse transfer function of the actuator to be summed up with the PID control signal. The theoretical analysis including the stability of the proposed AFC method has been sufficiently described in (Burdess \& Hewit 1986). A number of methods to estimate the mass have been proposed in previous studies such as through the use of artificial intelligence (AI) and crude approximation techniques (Burdess \& Hewit 1986). In this study, FL method is used to approximate the estimated mass. The main challenge of the AFC method is to acquire appropriate estimation of the mass needed to compute the disturbance $F_{\mathrm{d}}$ in the feedback loop. A conventional PID that is used with the AFC scheme can be typically represented by the following equation:

$$
G_{\mathrm{c}}(s)=K_{\mathrm{p}}+K_{\mathrm{i}} / s+K_{\mathrm{d}} s
$$

where $K_{\mathrm{p}}, K_{\mathrm{i}}$ and $K_{\mathrm{d}}$ are the proportional, integral and derivative gains respectively.

\section{Simulation}

MATLAB, Simulink and Control System Toolbox (CST) software are used to simulate the FIV model with the controllers. The actuators are assumed to be piezoelectric with hysteresis. They provide the necessary external energy to suppress vibration in the model. The parameters used in this study are taken from the previous works (Ouyang 2009; Utz von Wagner et al 2007). However, some need to be adapted to suit the application in the simulation. The detailed parameters are given as follows.

Mathematical model parameters:

- Body mass, $m=0.25 \mathrm{~kg}$

- Spring stiffness, $k=15000 \mathrm{~N} / \mathrm{m}, k_{1}=26250 \mathrm{~N} / \mathrm{m}, k_{2}=27000 \mathrm{~N} / \mathrm{m}$

- Damping coefficient, $c_{1}=1.49 \mathrm{Ns} / \mathrm{m}, c_{2}=1.5 \mathrm{Ns} / \mathrm{m}$

- Initial friction coefficient, $\mu=0.3$

- Normal pre-load, $N=100 \mathrm{~N}$

- Velocity of the belt, $v_{\mathrm{B}}=25 \mathrm{~km} / \mathrm{h}$

- Actuator gain, $Q_{1}=3, Q_{2}=1.5$

- Reference input $=0.0 \mathrm{~m}$ (i.e., no vibration).

In this work, several types of operating conditions are deliberately introduced to the system to evaluate the robustness of the control system. The Simulink diagram of the passive FIV system model is shown in figure 3. The schematic block diagram is constructed from equation (1). In order to have an active FIV system, two actuator forces for compensating the disturbances (that are actually inherent in the FIV system) are required. These forces are controlled by two individual PID controllers which typically involves two negative feedback loops. Hence, there are two inputs to the dynamic FIV system constituting the inputs to the actuator blocks. Figure 4 shows the active FIV system.

To obtain a better overall performance of the system, AFC is added to the pure PID scheme to effectively form a two DOF controller. The AFC Simulink diagram includes the estimated mass which is obtained by using FL (fuzzy logic) and the inverse function of the actuator. The input to the AFC control is the mass acceleration and the output is the estimated disturbance multiplied with the inverse transfer function of the actuator which is then summed with the PID controller output. In order to get the effective results using this method, it is required to acquire 


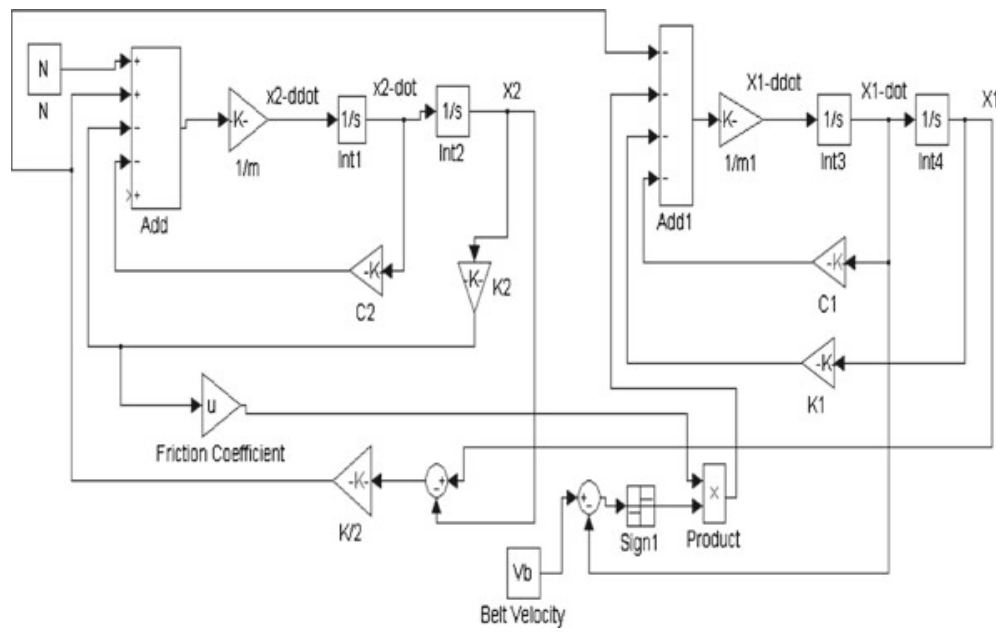

Figure 3. Passive FIV system.

an appropriate FL estimator to obtain a suitable value of the estimated mass combined with the best tuning of the PID controller gains. Figure 5 shows an AFC scheme used in the study to suppress the vibration and noise of the friction-induced vibration model that is mainly caused due to mode coupling in the system. It can be seen that each PID controller is equipped with an AFC loop that is equipped with FL estimators to evaluate the estimated masses.

As can be seen in figure 5, two actuators (assuming to be of piezoelectric type) are used for producing the actuator forces in the directions of $x_{1}$ and $x_{2}$. One of the most important factors that affect the behaviour of a piezoelectric actuator is hysteresis which usually occurs when the piezoelectric body is deformed. A part of the mechanical energy is stored as an elastic strain energy while the other is dissipated as heat during small internal sliding events. Hysteresis usually shows itself as an offset between the position path travelled during the application and removal of the excitation field. The size of the offset depends on many factors such as the material used or the field level and so on, the range of the offset varies from $1 \%$ to $10 \%$ (Piezoelectric Motor/Actuator kit 2007). In some cases, this percentage might be higher such as in low voltage

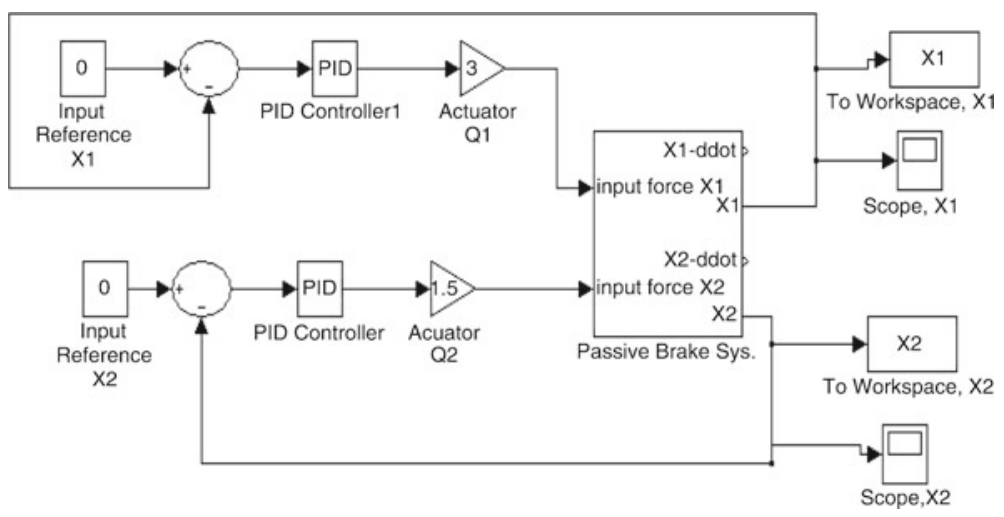

Figure 4. Active FIV system. 


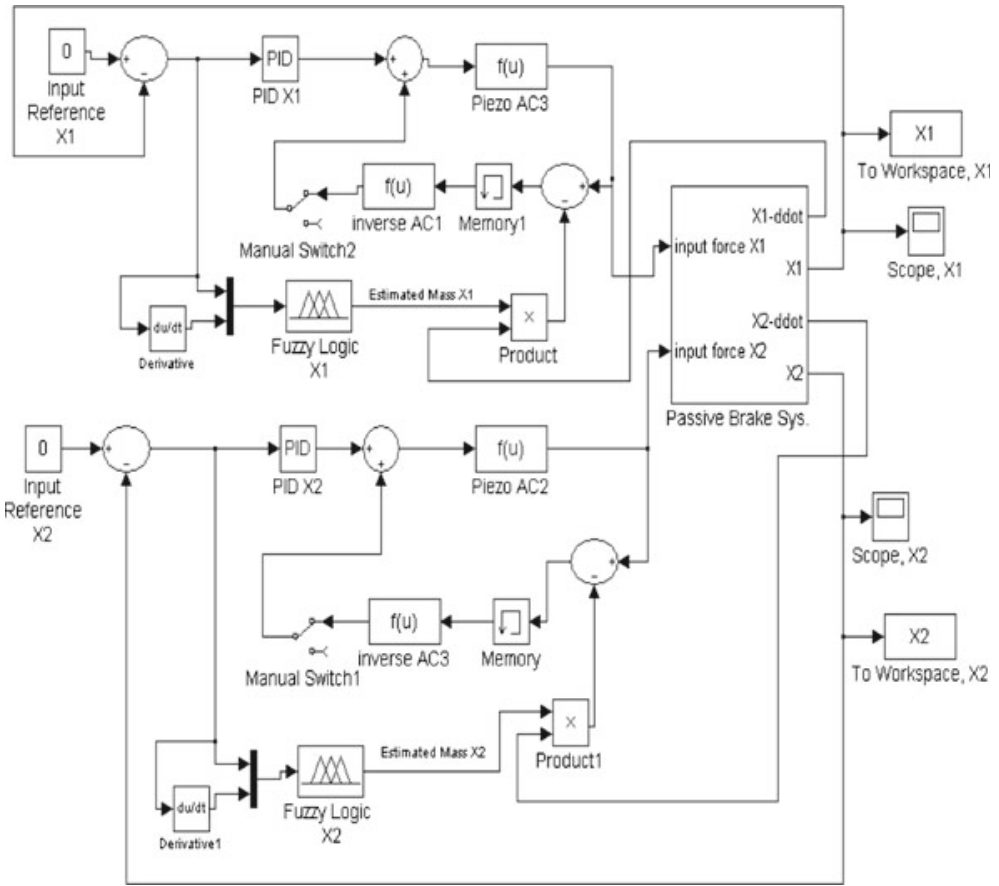

Figure 5. Simulink diagram of the proposed AFC model with fuzzy logic estimators and piezoelectric actuators.

stack piezoelectric actuators which have $15 \%$ hysteresis during their operation (Low voltage Piezoelectric Stacks 2007). Figure 6 shows the hysteresis and creep behaviour of a piezoelectric element (Piezoelectric Motor/Actuator kit manual 2007).

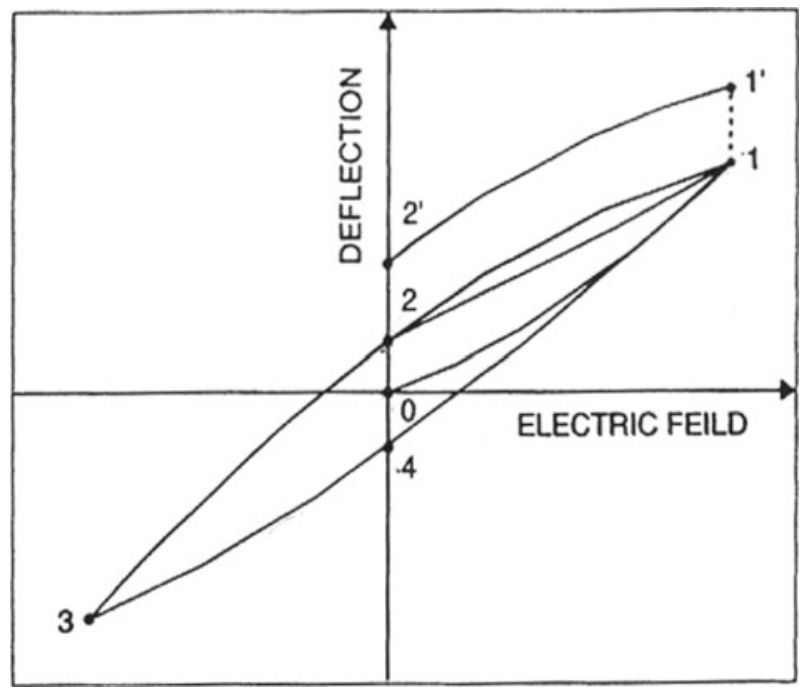

Figure 6. Typical hysteresis and creep behaviour of piezoelectric element (Piezoelectric Motor/Actuator kit 2007). 


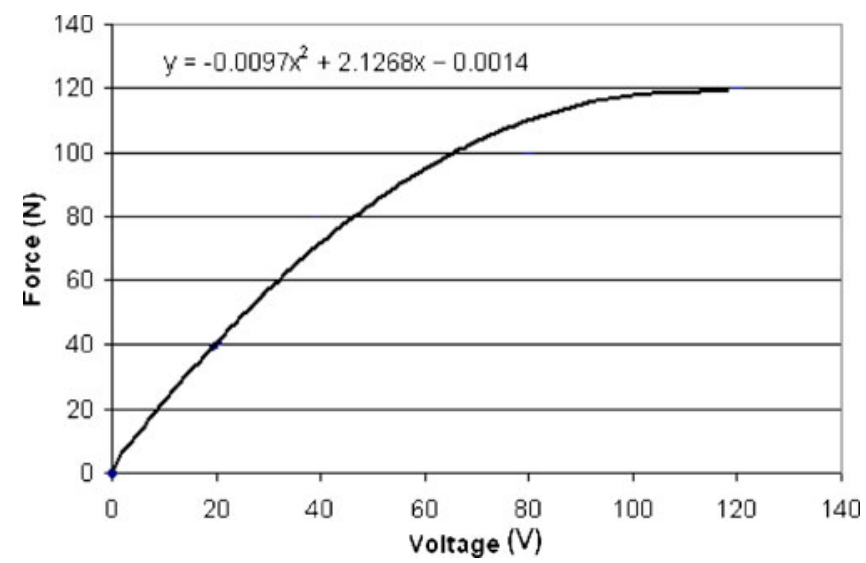

Figure 7. The simulated hysteresis effect of the piezoelectric actuator.

When the element is energized, it will move to position 1, then after de-energizing it will remove to position 2, and if it is re-energized immediately, it will again follow path 2-1. If the element is left energized for a long time, it will start to creep and thus follow path 1-1'. When it is de-energized, it will follow path 1'-2', then after a long time of being at rest, it will revert to position 0. In case the element is using alternating current (AC) mode, it will follow 1-2-3-4-1 (Piezoelectric Motor/Actuator kit 2007).

Thus, in order to reduce the vibrations with the piezoelectric actuator it is required to consider path 1-2 that is shown in figure 6 , because the actuator is in a charging and discharging loop during its operation.

By using MATLAB and Microsoft Excel, the nonlinear hysteresis equation of path 2-1 for the considered piezoelectric actuators is simulated, the graph of this simulation is shown in figure 7 and the obtained equation is written on the graph.

Since in the AFC loop it is required to have the inverse function of the actuator, again by using MATLAB and Microsoft Excel, the approximate inverse function of the simulated actuator

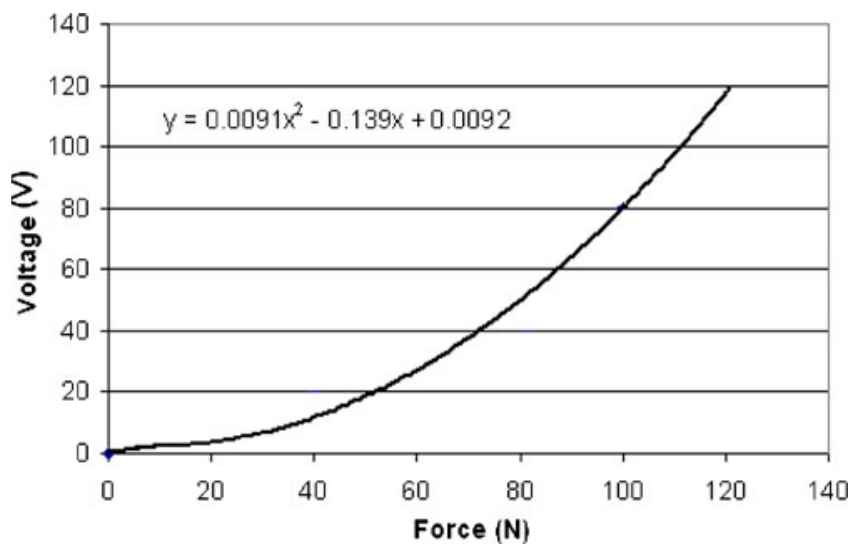

Figure 8. The inverse function of the simulated piezoelectric actuator. 
Table 1. The values for the PID parameters.

\begin{tabular}{llll}
\hline PID parameters & $K_{\mathrm{p}}$ & $K_{\mathrm{i}}$ & $K_{\mathrm{d}}$ \\
\hline PID $x_{1}$ & 3.5 & 1.5 & 6 \\
PID $x_{2}$ & 0.5 & 0.02 & 0.08 \\
\hline
\end{tabular}

is obtained. Figure 8 shows the approximated inverse function of the simulated piezoelectric actuator.

To tune the PID controllers, Ziegler-Nichol's method is used and the results are tabulated in table 1. In order to have a better performance of the PID controller, fuzzy logic can be implemented to obtain the required values (Pratihar et al 1999; Rajendra \& Pratihar 2011). But in this study considering the values constant and obtaining them by Ziegler-Nichol's method is sufficient, also because of requiring more computation and thus causing time delay constant values are considered.

After simulating the piezoelectric actuator, it is required to design two FL estimators (with using MATLAB Fuzzy Logic Toolbox) to obtain suitable values of the estimated masses in the two AFC loops. The FL estimators are designed by considering two inputs and one output, in which the inputs to the fuzzy estimator are the error and the error rate that are obtained during the control process (as shown in figure 5) and the output is the required estimated mass for the AFC loop. The range of the errors and estimated mass for the fuzzification part is mainly obtained from previous simulation results and intuition-based from past experience. The triangular-shaped membership functions are chosen due to its popularity and effectiveness. Figures 9 and 10 show the inputs of the FL estimator (in the direction of $x_{1}$ ). It can be seen that the range of the error is limited from -0.2 to 0.2 and five triangular membership functions are used starting from very low, low, medium, high and very high. The range of the error rate is limited from -8 to 8 and three membership functions are used which start from low, medium and high.

Figure 11 shows the output, i.e., the estimated mass of the FL estimator (in the direction of $x_{1}$ ). It can be seen that the range of the output is limited from 0.11 to 0.21 and five triangular membership functions are used and similar to the input, they start from very low to very high.

The rules that are defined in the fuzzy logic estimator for direction $\mathrm{X}_{1}$ are as listed below.

1. If (error X1 is very low) and (error rate $\mathrm{X} 1$ is low), then (estimated mass is very low).

2. If (error $\mathrm{X} 1$ is very low) and (error rate $\mathrm{X} 1$ is medium), then (estimated mass is very low).

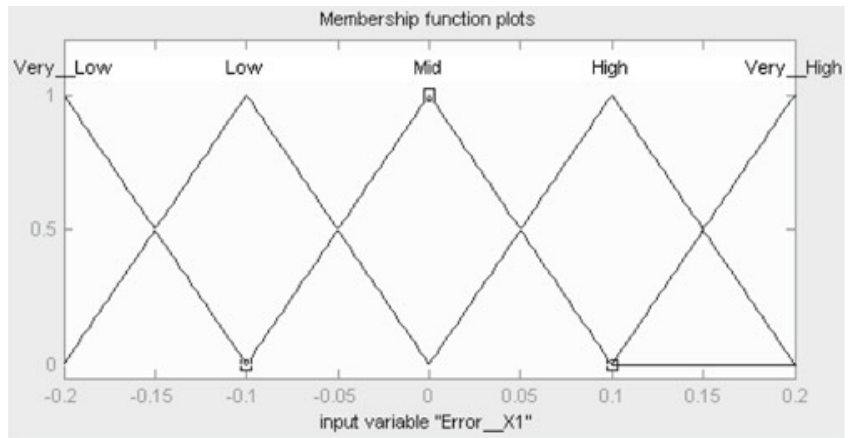

Figure 9. Error input of the FL estimator in the direction of $x_{1}$. 


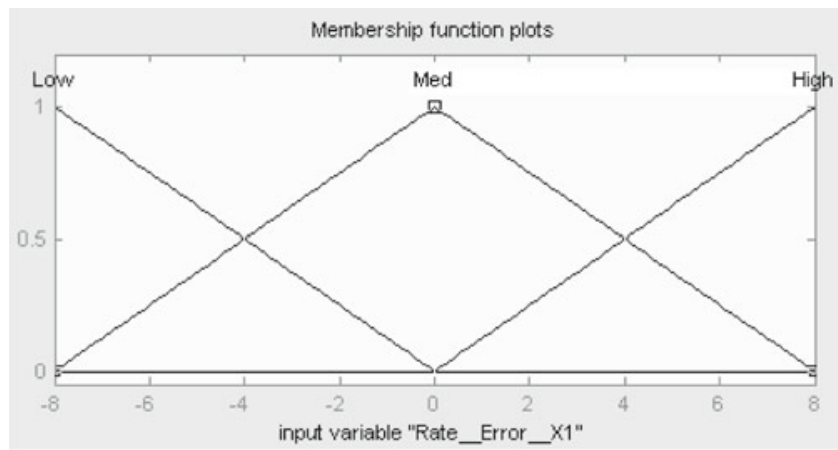

Figure 10. Error rate input of the FL estimator in the direction of $x_{1}$.

3. If (error X1 is very low) and (error rate $\mathrm{X} 1$ is high), then (estimated mass is low).

4. If (error X1 is low) and (error rate X1 is low), then (estimated mass is very low).

5. If (error $\mathrm{X} 1$ is low) and (error rate $\mathrm{X} 1$ is medium), then (estimated mass is low).

6. If (error X1 is low) and (error rate X1 is high), then (estimated mass is medium).

7. If (error $\mathrm{X} 1$ is medium) and (error rate $\mathrm{X} 1$ is low), then (estimated mass is low).

8. If (error $\mathrm{X} 1$ is medium) and (error rate $\mathrm{X} 1$ is medium), then (estimated mass is medium).

9. If (error $\mathrm{X} 1$ is medium) and (error rate $\mathrm{X} 1$ is high), then (estimated mass is high).

10. If (error $\mathrm{X} 1$ is high) and (error rate $\mathrm{X} 1$ is low), then (estimated mass is medium).

11. If (error $\mathrm{X} 1$ is high) and (error rate $\mathrm{X} 1$ is medium), then (estimated mass is high).

12. If (error $\mathrm{X} 1$ is high) and (error rate $\mathrm{X} 1$ is high), then (estimated mass is very high).

13. If (error $\mathrm{X} 1$ is very high) and (error rate $\mathrm{X} 1$ is low), then (estimated mass is high).

14. If (error $\mathrm{X} 1$ is very high) and (error rate $\mathrm{X} 1$ is medium), then (estimated mass is very high).

15. If (error X1 is very high) and (error rate $\mathrm{X} 1$ is high), then (estimated mass is very high).

The fuzzy inference system (FIS) considered is chosen to be of Mamdani type and the centroidal method is used for defuzzification. Figure 12 shows a graphical representation of the fuzzy logic estimator in direction $x_{1}$ which is obtained by surface viewer from the MATLAB Fuzzy Logic Toolbox.

The same process is exactly applied to design the FL estimator in the direction of $x_{2}$, in which the error and the error rate are considered as the inputs and the output of the fuzzy controller is

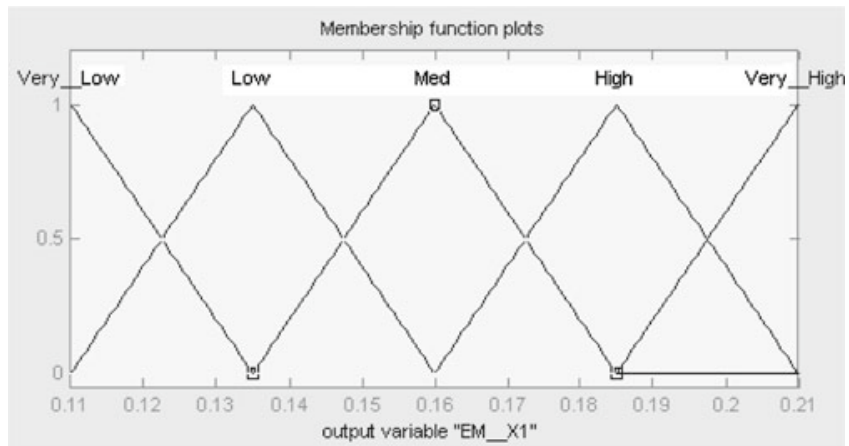

Figure 11. Output of the FL estimator in the direction of $x_{1}$. 


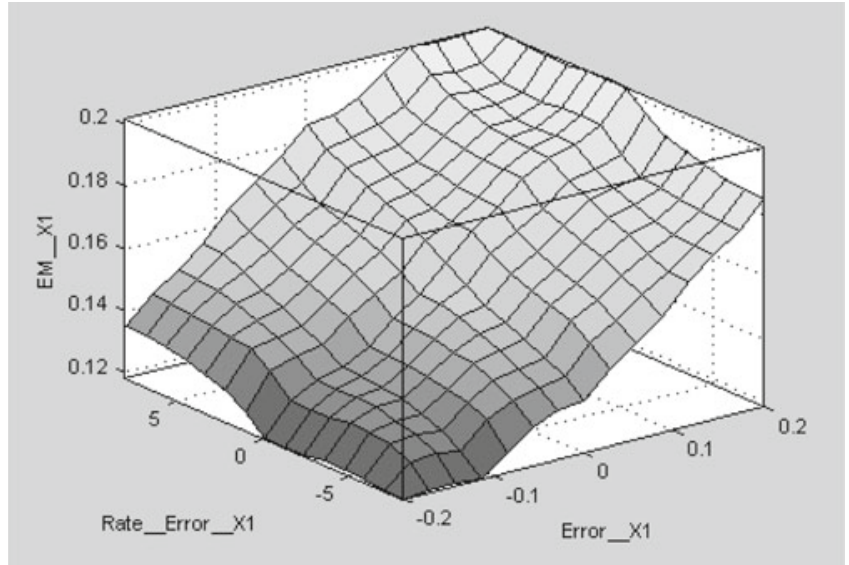

Figure 12. Graphical view of the FL estimator in the direction of $x_{1}$.

the required estimated mass. The range of the error that is defined to the input is [-0.08 0.08$]$, and five triangular membership functions are used as shown in figure 13, the range of the error rate is between [ -30 and 30] and again tree membership functions are used starting from low to high as shown in figure 14. The output range of the fuzzy controller is set to [0.12 0.2]. Again, five triangular membership functions are implemented. Figure 15 shows the output membership functions.

The rules that are defined in the fuzzy logic estimator for direction $\mathrm{X}_{2}$ are as listed below.

1. If (error X2 is very low) and (error rate X2 is low), then (estimated mass is very low).

2. If (error $\mathrm{X} 2$ is very low) and (error rate $\mathrm{X} 2$ is medium), then (estimated mass is very low).

3. If (error X2 is very low) and (error rate X2 is high), then (estimated mass is low).

4. If (error X2 is low) and (error rate X2 is low), then (estimated mass is very low).

5. If (error X2 is low) and (error rate X2 is medium), then (estimated mass is low).

6. If (error X2 is low) and (error rate $\mathrm{X} 2$ is high), then (estimated mass is medium).

7. If (error $\mathrm{X} 2$ is medium) and (error rate $\mathrm{X} 2$ is low), then (estimated mass is low).

8. If (error $\mathrm{X} 2$ is medium) and (error rate $\mathrm{X} 2$ is medium), then (estimated mass is medium).

9. If (error X2 is medium) and (error rate X2 is high), then (estimated mass is high).

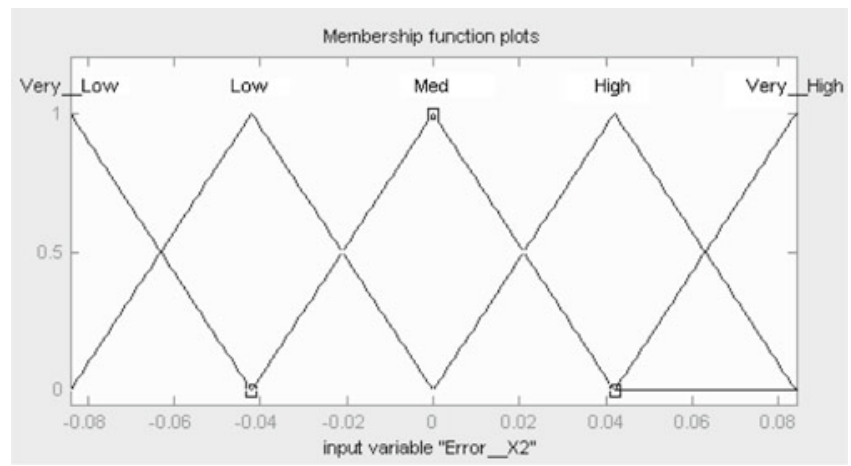

Figure 13. Error input of the FL estimator in the direction of $x_{2}$. 


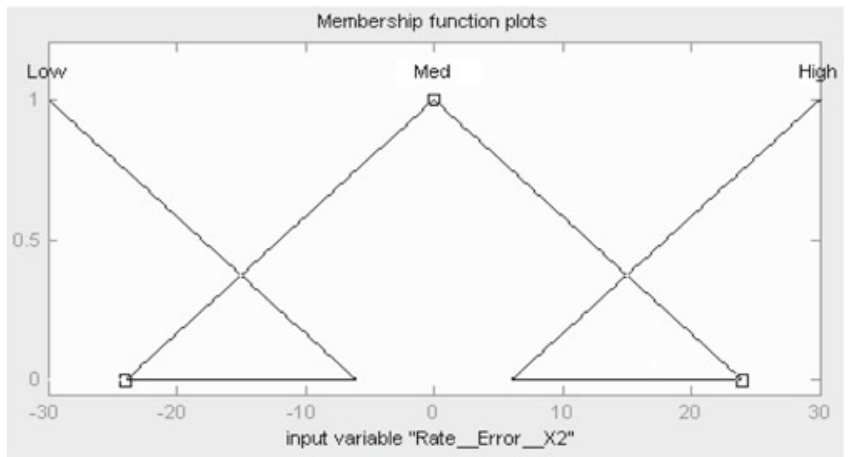

Figure 14. Error rate input of the FL estimator in the direction of $x_{2}$.

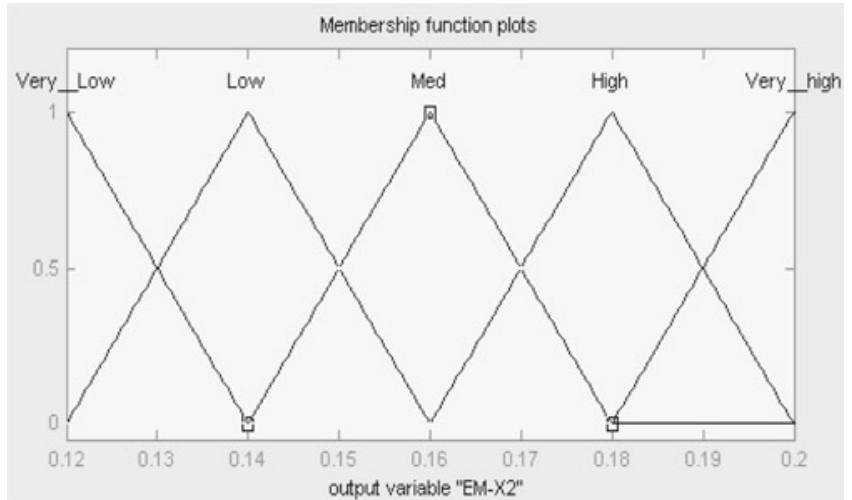

Figure 15. Output of the FL estimator in the direction of $x_{2}$.

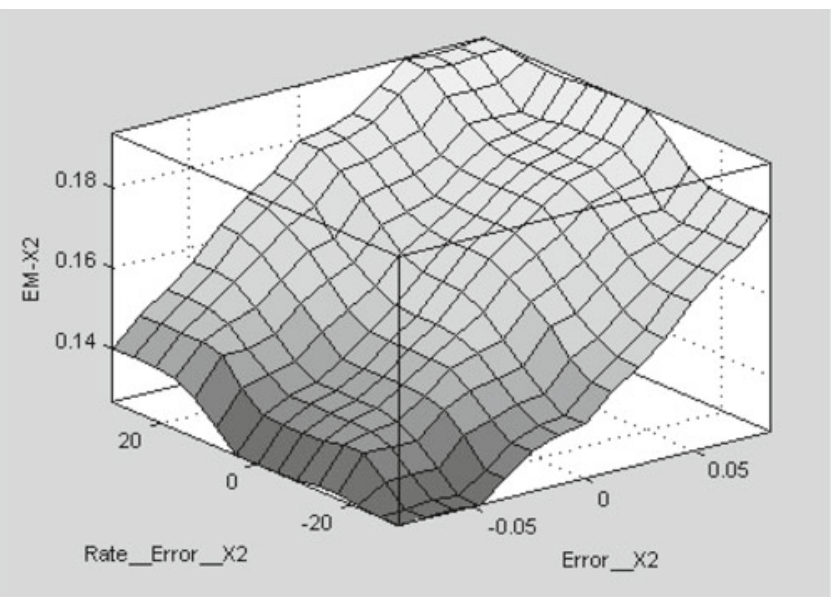

Figure 16. Graphical view of the FL estimator in the direction of $x_{2}$. 


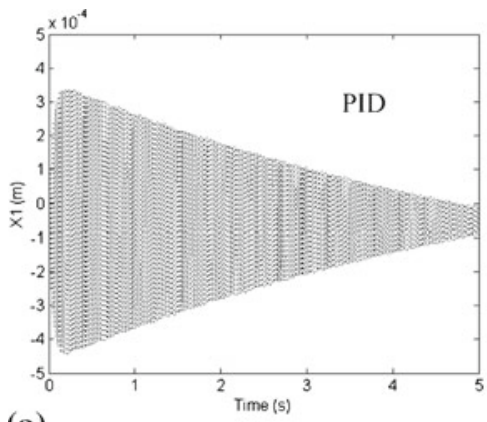

(a)

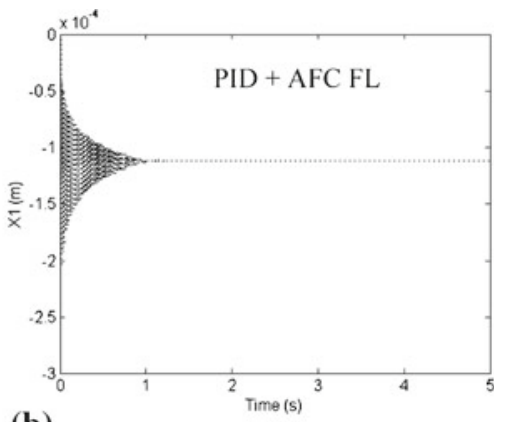

(b)

Figure 17. Response of the direction $x_{1}$ in the FIV model having the friction coefficient: $\mu=0.3$. (a) PID, (b) PID+AFC FL.

10. If (error $\mathrm{X} 2$ is high) and (error rate $\mathrm{X} 2$ is low), then (estimated mass is medium).

11. If (error $X 2$ is high) and (error rate $X 2$ is medium), then (estimated mass is high).

12. If (error $X 2$ is high) and (error rate $X 2$ is high), then (estimated mass is very high).

13. If (error $\mathrm{X} 2$ is very high) and (error rate $\mathrm{X} 2$ is low), then (estimated mass is high).

14. If (error $\mathrm{X} 2$ is very high) and (error rate $\mathrm{X} 2$ is medium), then (estimated mass is very high).

15. If (error $\mathrm{X} 2$ is very high) and (error rate $\mathrm{X} 2$ is high), then (estimated mass is very high).

Figure 16 shows a graphical representation of the FL estimator in the direction of $x_{2}$.

\section{Results and discussion}

At first, the simulation is performed under normal condition, in which the initial coefficient of friction was, $\mu=0.3$. The simulation is performed without using AFC and only the pure PID controller is applied. The result of this simulation can be seen in figures 17 and 18. It is shown that the vibration level in both directions is relatively high and reducing by time, but when the simulation is carried out with considering the PID controller plus 100\% AFC engaged (switched on), much better reduction in vibration is observed.

Responses in the frequency domain are also obtained in directions $x_{1}$ and $x_{2}$ as shown in figures 19a and b and 20a and b, respectively.
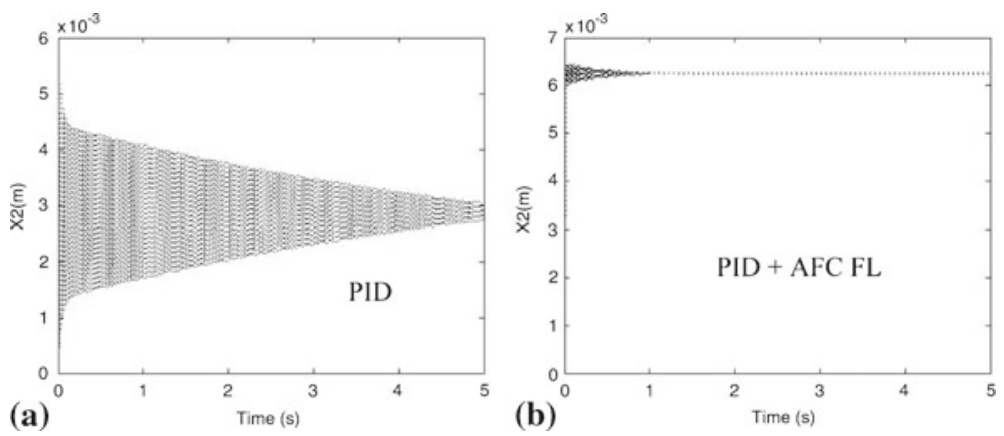

Figure 18. Response of the direction $x_{2}$ in the FIV model having the friction coefficient: $\mu=0.3$. (a) PID, (b) PID+AFC FL. 

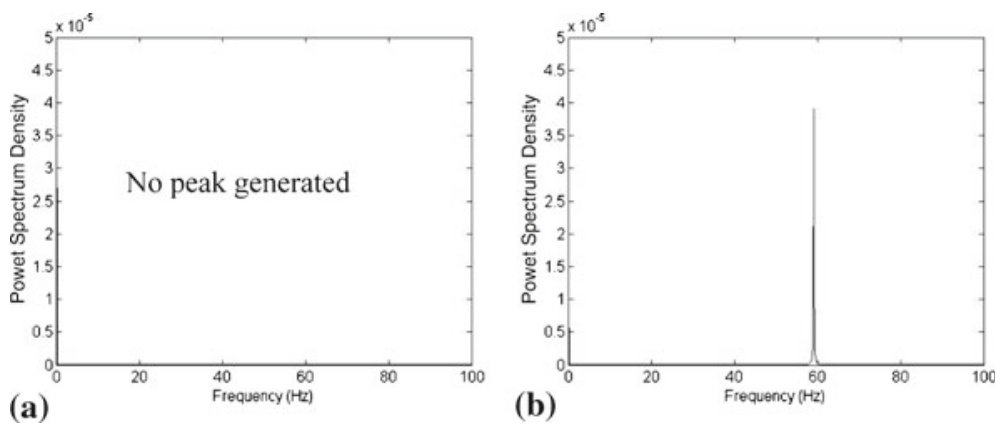

(b)

Figure 19. Results in frequency domain in the direction of $x_{1}$ with $\mu=0.3$ for (a) PID+AFC, (b) PID schemes.

The minimum value of $\mu$ that makes the system unstable is known as the critical friction coefficient, $\mu_{\mathrm{cr}}$. By trial and error or crude approximation, it is found that this value is 0.302 , where from this value of friction coefficient the PID controllers are unable to stabilize the FIV system.

The next simulation is performed with the friction coefficient of 0.305 which is more than the value of $\mu_{\mathrm{cr}}$. It can be seen from figures 21 and 22 that when the FIV system has only PID controllers, the response is very unstable and the vibration swiftly increases with time, meaning unstable system. However, the stability of the system is considerably improved when the PID controller is integrated with AFC element. The results in frequency domain are shown in figures 23 and 24.

The last simulation is to increase the friction coefficient to 0.35 , which is very much higher than the friction coefficient values that are presented. By running the simulation, it is noticed by the results that are shown in figures 25 and 26 that the vibrations have very unstable and the PID controllers are unable to suppress them. However, after engaging AFC to the PID controllers the vibration in the system is reduced very much to a noticeable amount.

The frequency response of the brake model operating with a friction coefficient of 0.35 is shown in figures 27 and 28.
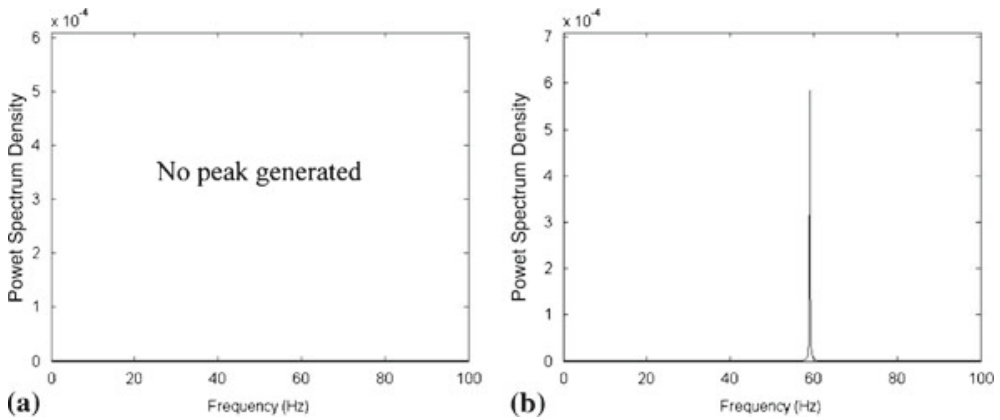

Figure 20. Results in frequency domain in the direction of $x_{2}$ with $\mu=0.3$ for (a) PID+AFC, (b) PID schemes. 

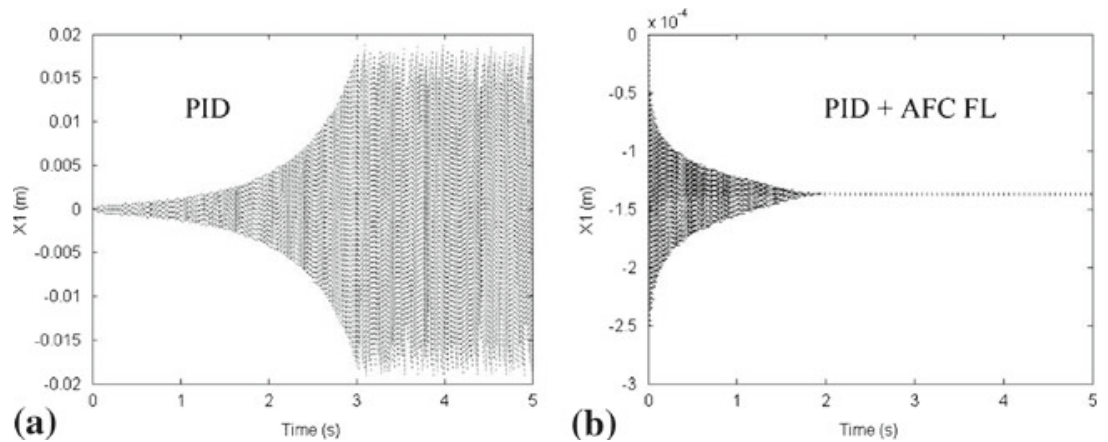

Figure 21. Response of the direction $x_{1}$ in the FIV model having the friction coefficient: $\mu=0.305$ (a) PID, (b) PID+AFC FL.
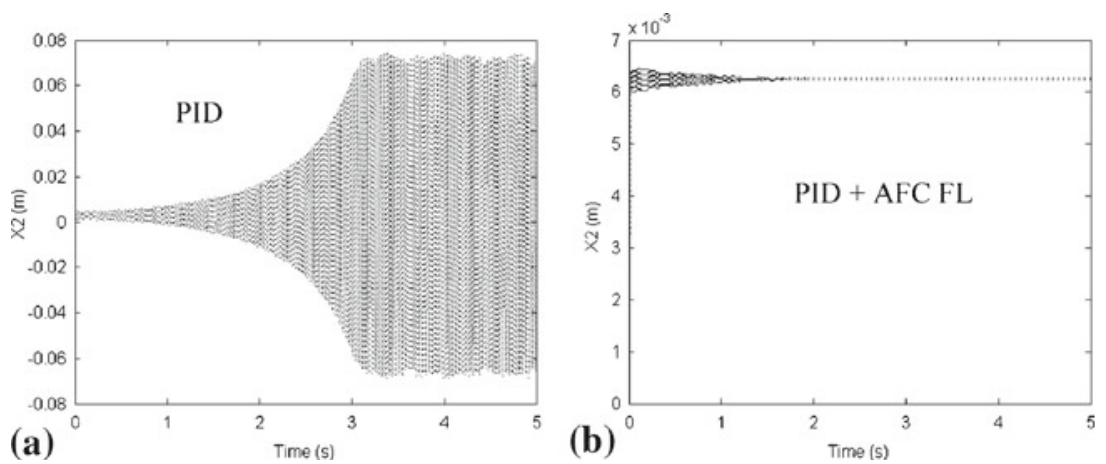

Figure 22. Response of the direction $x_{2}$ in the FIV model friction coefficient: $\mu=0.305$ (a) PID, (b) PID+AFC FL.
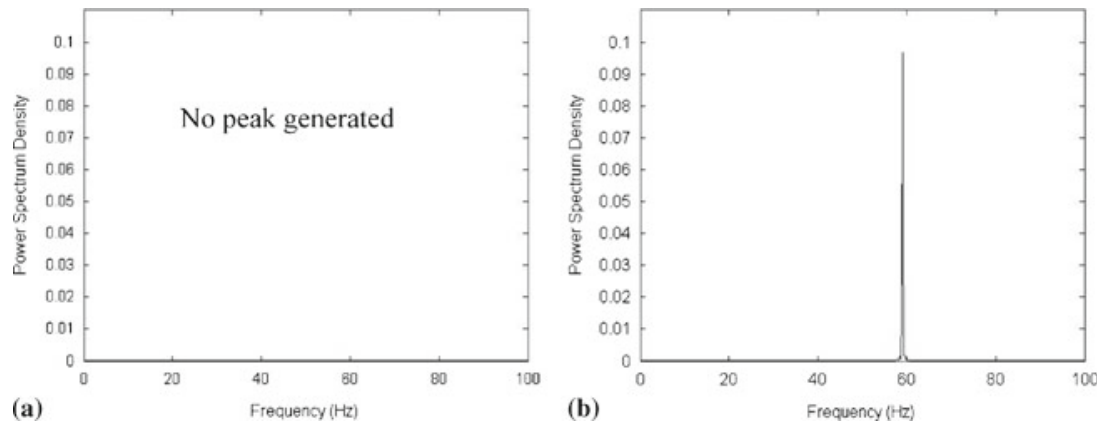

Figure 23. Results in frequency domain in the direction of $x_{1}$ with $\mu=0.305$ for (a) PID+AFC FL, (b) PID schemes. 

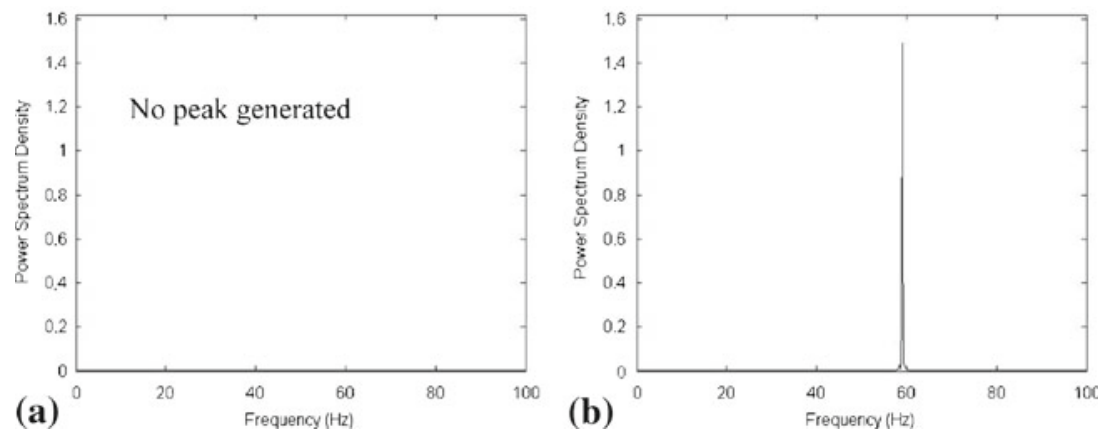

Figure 24. Results in frequency domain in the direction of $x_{2}$ with $\mu=0.305$ for (a) PID+AFC FL, (b) PID schemes.
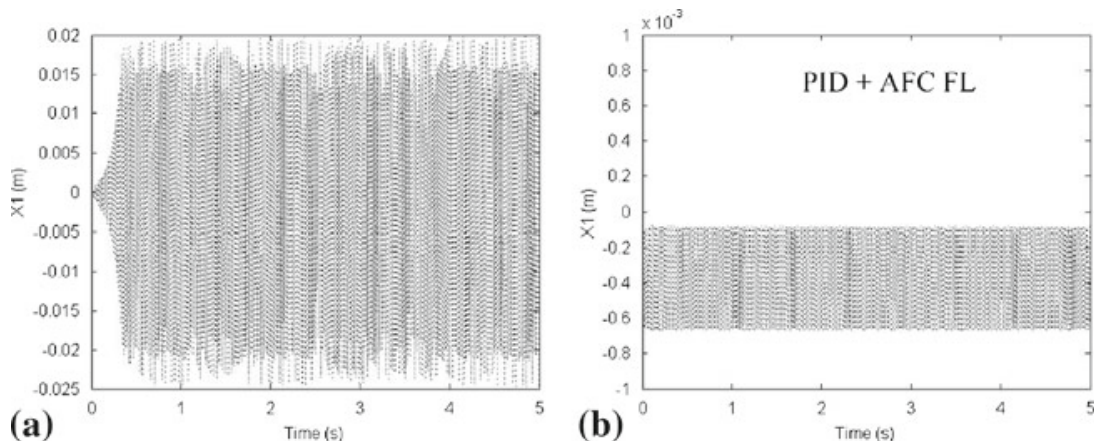

Figure 25. Response of the direction $x_{1}$ in the FIV model having the friction coefficient: $\mu=0.305$ (a) PID, (b) PID+AFC FL.
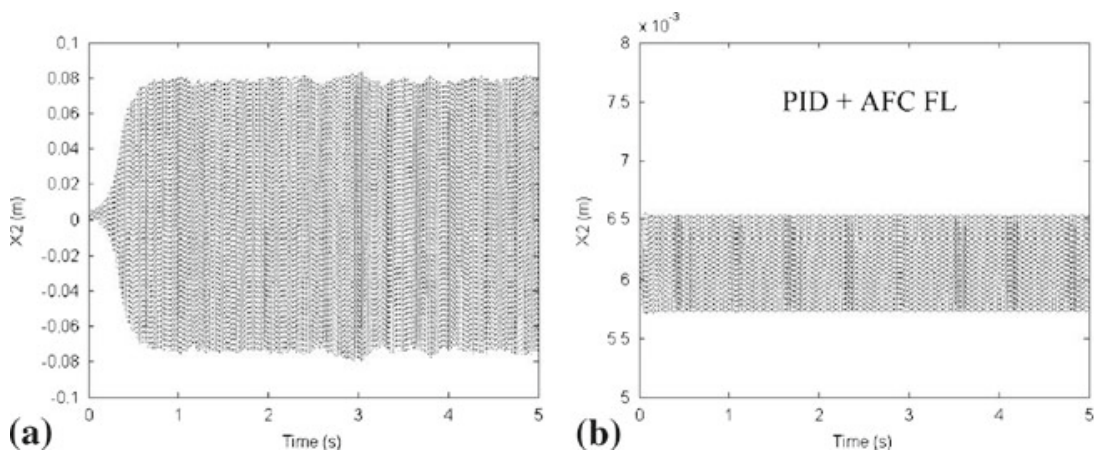

Figure 26. Response of the direction $x_{2}$ in the FIV model having the friction coefficient: $\mu=0.305$ (a) PID, (b) PID+AFC FL. 

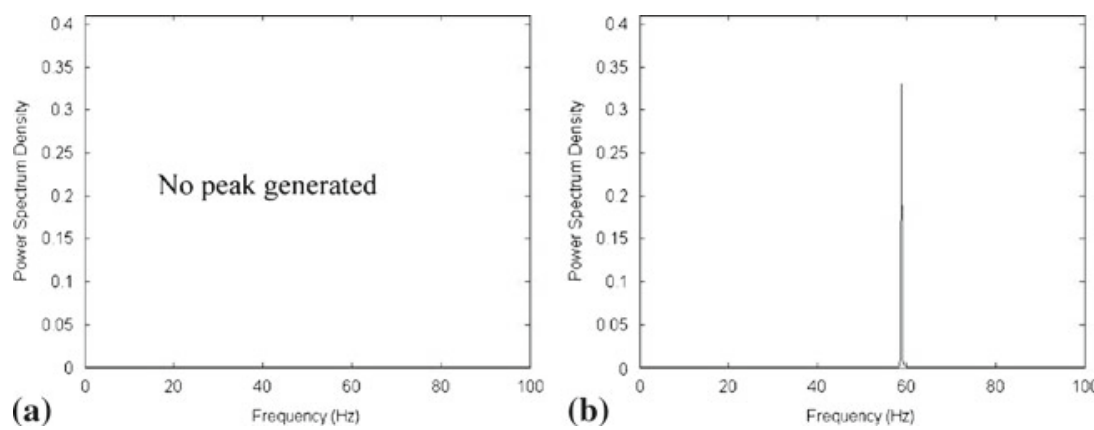

Figure 27. Results in frequency domain in the direction of $x_{1}$ with $\mu=0.35$ for (a) PID+AFC, (b) PID schemes.

At this condition in which the friction coefficient is 0.35 (one of the worst conditions), the relative magnitude between the applied preload and the load exerted by the actuator number two is estimated. The reason for actuator number two is chosen because it controls the vertical direction which is more critical. The results show that when the preload $N$ is $100 \mathrm{~N}$ the maximum force that is applied by the actuator is $117 \mathrm{~N}$, and when the preload is reduced to $50 \mathrm{~N}$ the maximum force that is applied by the actuator is $25 \mathrm{~N}$, the same amount is obtained when the preload force is increased to $150 \mathrm{~N}$. Thus, it can be understood that when the preload force $N$ is increased or reduced from $100 \mathrm{~N}$, the force that is applied by the actuator reduces noticeably. Somehow, this shows that when the preload force is $100 \mathrm{~N}$, the friction-induced vibration system is in one of its most critical conditions and that the actuators are applying the highest force magnitude and the AFC system is capable of reducing the unwanted vibrations.

The dissipated energy is evaluated in the cases in which the AFC is engaged and when the system is operating without any controller and actuator applied to it, the energy equation is:

$$
\text { Dissipated Energy }=\text { Friction Force } \times \text { Sliding Velocity } \text {. }
$$

The maximum amount of dissipated energy when the system is operating without a control system is $10 \mathrm{~kJ}$, and the maximum amount of the dissipated energy when AFC is fully engaged is around $0.5 \mathrm{~kJ}$. This means that the dissipated energy is reduced very much when the unwanted noise or vibrations reduce. In vehicles, the objective is to maintain the braking efficiency while
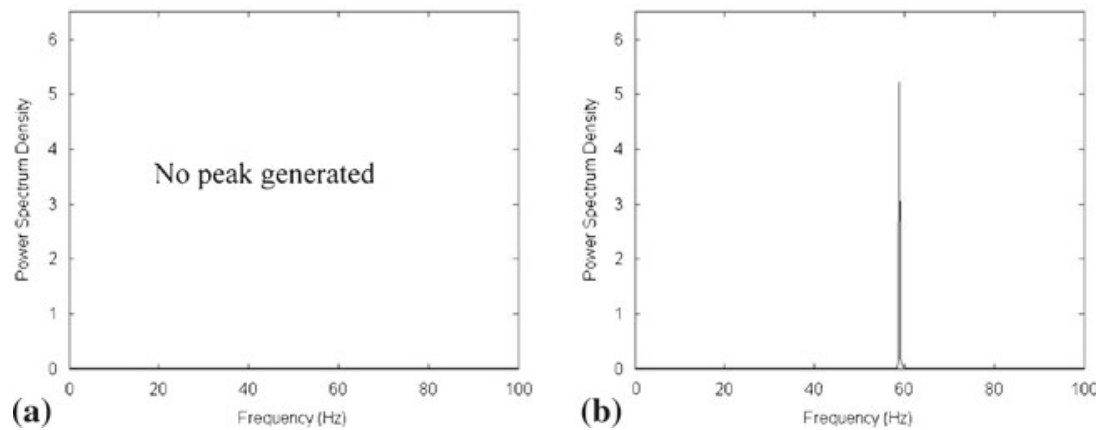

Figure 28. Results in frequency domain in the direction of $x_{2}$ with $\mu=0.35$ for (a) PID+AFC, (b) PID schemes. 
reducing noise and vibrations. Since the dissipated energy is reducing, the efficiency of the braking system is also increased, due to that reason the overall efficiency is obtained by:

$$
\text { Overall Efficiency }=(\text { Brake Force }) /(\text { Vehicle Weight }) \times 100 \% \text {. }
$$

Depending on the type of the vehicle, due to the reason that the actuators increase the brake force and reduce the dissipated energy the overall efficiency will increase.

\section{Conclusion}

From this study, a newly proposed AFC-based scheme equipped with FL estimators and considering the use of piezoelectric actuators with hysteresis has been shown to reduce the vibration that is caused by friction-induced vibration due to mode coupling. According to the simulation results, it is obvious that when a pure PID controller is applied to the friction-induced vibration system, vibration and noise are in fact gradually reduced but still a noticeable amount of them remain especially in the first two seconds of the simulation period. In most cases, by changing the operating conditions, the PID controller unable to reduce the vibration at all and instead, it leads the system towards instability. However, upon applying the AFC-based technique, the vibration and noise are considerably reduced and almost approaching a zero datum, thereby implying the effectiveness of the proposed strategy in countering the adverse conditions. For future work, it is planned to design an experimental rig-based on the theoretical settings through the simulation study.

\section{References}

Abendroth H and Wernitz B 2000 The integrated test concept: Dyno-vehicle, performance-noise, Technical Report 2000-01-2774, SAE, Warrendale, PA

Akay A, Wickert J and Xu Z 2000 Investigating mode lock-in and friction interface, Final Research Report, Carnegie Mellon University, pp. 1-52

Burdess J S and Hewit J R 1986 An active method for the control of mechanical systems in the presence of unmeasurable forcing. Mech. Mach. Theory 21(5): 393-400

Chatterjee S and Mahata P 2009 Time-delayed absorber for controlling friction-driven vibration. J. Sound Vib. 322: 39-59

Earles S W E and Lee C K 1976 Instability arising from the friction interaction of a pin-disc system resulting in noise generation. ASME J. Eng. Ind. 98: 81-86

Fieldhouse J D and Newcomb P 1993 The application of holographic interferometry to the study of disc brake noise, S.A.E. Technical Paper 930805

Hashemi-Dehkordi S M, Mailah M and Abu Bakar A R 2009a Intelligent active force control with piezoelectric actuators to reduce friction induced vibration due to negative damping. Int. Rev. Electr. Eng. (IREE) 4(6): 1294-1305

Hashemi-Dehkordi S M, Mailah M and Abu Bakar A R 2009b Implementation of active force control to disk brake noise-free performance. Int. Mech. Eng. 3(4): 481-488

Hashemi-Dehkordi S M, Mailah M and Abu-Bakar A R 2010 Suppressing friction-induced vibration due to negative damping and mode coupling effects using active force control. Aust. J. Basic App. Sci. 4(8): $3917-3933$

Hewit J R and Burdess J S 1981 Fast dynamic decoupled control for robotics using active force control. Mech. Mach. Theory 16(5): 535-542

Hoffmann N, Bieser S and Gaul L 2004 Harmonic balance and averaging techniques for stick-slip limitcycle determination in mode-coupling friction self-excited systems. Technische Mechanik 24: 185 
Kinkaid N M, O'Reilly O M and Papadopoulos P 2003 Review: automotive disc brake squeal. J. Sound Vib. 267: 105-166

Low Voltage Piezoelectric Stacks 2007 Catalog \#7B, Piezo systems, Inc., Woburn, MA 01801, USA

Mailah M and Rahim N I A 2000 Intelligent active force control of a robot arm using fuzzy logic, in: Proceedings of IEEE International Conference on Intelligent Systems and Technologies, Kuala Lumpur, Malaysia, 2: 291-296

Nakano K and Maegawa S 2009 Safety-design criteria of sliding systems for preventing friction-induced vibration. J. Sound Vib. 324(3-5): 539-555

Ouyang H 2009 Prediction and assignment of latent roots of damped asymmetric systems by structural modifications. Mech. Syst. Signal Process. 23: 1920-1930

Ouyang H, Mottershead J E, Cartmell M P and Friswell M I 1998 Friction-induced parametric resonances in discs: effect of a negative friction-velocity relationship. J. Sound Vib. 209(2): 251-264

Ouyang H, Baeza L and Hu S 2009 A receptance-based method for predicting latent roots and critical points in friction-induced vibration problems of asymmetric systems. J. Sound Vib. 321: 1058-1068

Papinniemi A, Lai J C S, Zhao J and Loader L 2002 Brake squeal: a literature review. Appl. Acoust. 63: 391-400

Piezoelectric Motor/Actuator kit manual 2007 Piezo systems, Inc., Woburn, MA 01801, USA

Pratihar D K, Deb K and Ghosh A 1999 A genetic-fuzzy approach for mobile robot navigation among moving obstacles. Int. J. Prox. Reason. 20: 145-172

Priyandoko G, Mailah M and Jamaluddin H 2009 Vehicle active suspension system using skyhook adaptive neuro active force control. Mech. Syst. Signal Process. 23(3): 855-868

Rajendra R and Pratihar D K 2011 Particle swarm optimization vs. genetic algorithm to develop integrated scheme for obtaining optimal mechanical structure and adaptive controller of a robot. Intell. Control. Autom. 2: 430-449

Shin K, Brennan M J, Joe Y-G and Oh J-E 2004 Simple models to investigate the effect of velocity dependent friction on the disk brake squeal noise. Int. J. Automot. Technol. 5(1): 61-67

U-Xuan Tan, Win Tun Latt, Ferdinan Widjaja, Cheng Yap Shee, Cameron N. Riviere, Wei Tech Ang 2009 Tracking control of hysteretic piezoelectric actuator using adaptive rate-dependent controller. Sensors Actuators A 150: 116-123

Utz von Wagner, Hochlenert D and Hagedorn P 2007 Minimal models for disk brake squeal. J. Sound Vib. 302: $527-539$ 